\title{
ANÁLISE DOS FUNDAMENTOS DA TEORIA BASEADA NOS RECURSOS
}

\section{RESUMO}

Frequentemente as hipóteses da teoria baseada nos recursos (RBV) são testadas utilizando uma abordagem centrada na heterogeneidade dos recursos, permitindo apenas identificar que recursos ou capacidades específicas devem ser exploradas para alcançar a vantagem competitiva. Neste artigo, utilizamos uma abordagem conceptual procurando testar se as combinações de recursos e capacidades com valor, raridade e inimitabilidade, mais do que a sua especificidade, determinam maior vantagem competitiva e performance. Os resultados obtidos de 368 questionários validados por gestores de empresas de vários setores económicos portugueses corroboram as hipóteses de que, o aumento do valor, da raridade e da inimitabilidade das combinações testadas eleva a vantagem competitiva da empresa e, que a performance é alcançada por esta via. As conclusões são de interesse para o desenvolvimento da investigação no campo da gestão estratégica e, para os gestores possibilitando-lhes a tomada de decisões estratégicas com base no valor, raridade e inimitabilidade das combinações de recursos com capacidades da sua empresa.

Palavras-chave: Valor; Raridade; Inimitabilidade; Vantagem Competitiva; Performance.

ANALYSIS OF FOUNDATIONS OF RESOURCE-BASED THEORY

\begin{abstract}
The assumptions of the resource-based theory (RBV) are often tested using an approach centered on the heterogeneity of resources, allowing only identify specific resources or capabilities that should be exploited to achieve competitive advantage. In this paper we use a conceptual approach seeking to test whether value, rare and inimitable combinations of resources and capabilities, rather than their specificity, achieve competitive advantage and performance. The results obtained from 368 questionnaires validated by business managers of several Portuguese economic sectors show that the increase in value, rarity and inimitability of the combinations tested, raises the company's competitive advantage, and that performance is achieved by this route. The findings are of interest for the development of the field of strategic management and for managers enabling strategic decision-making based on valuable, rare and inimitable company`s resources and capabilities combinations.
\end{abstract}

Keywords: Value; Rarity; Inimitability; Competitive Advantage; Performance. 


\section{ANÁLISIS DE FUNDAMENTOS DE TEORÍA DE RECURSOS BASADO}

\section{RESUMEN}

A menudo se corroboran las hipótesis de la teoría basada en los recursos (RBV) utilizando un enfoque centrado en la heterogeneidad de los mismos que, sólo permite identificar qué recursos o capacidades específicas deben explotarse para lograr la ventaja competitiva. En este artículo, utilizamos un enfoque conceptual buscando probar si, las combinaciones de recursos con capacidades de valor, escassez e inimitabilidad, más que su especificidad, determinan una mayor ventaja competitiva y un mayor rendimiento. Los resultados obtenidos de 368 encuestas validadas por los directivos de las empresas de diversos sectores económicos portugueses, corroboraron que el aumento del valor, escassez y de la inimitabilidad de las combinaciones probadas, aumenta la ventaja competitiva de la empresa y, que el rendimiento se obtiene de esta manera. Las conclusiones son de interés para el desarrollo de la investigación en el campo de gestión estratégica y para los diretivos empresariales permitindolles tomar decisiones estratégicas basadas en el valor, escassez y la inimitabilidad de las combinaciones de recursos con las capacidades de sus empresas.

Palabras-clave: Valor; Rendimiento; Ventaja Competitiva; Escassez; Inimitabilidad.

António José Pinto Pedrosa ${ }^{1}$ Fernando Manuel Pereira Oliveira Carvalho ${ }^{2}$

\footnotetext{
${ }^{1}$ Mestre em Estratégia Empresarial pela Universidade de Coimbra, Portugal. Professor do Instituto Politécnico de Leiria, Portugal. Portugal. E-mail: antonio.pedrosa@ipleiria.pt

${ }^{2}$ Doutor. Professor da Faculdade de Economia da Universidade de Coimbra, Portugal. Portugal. E-mail: $\underline{\mathbf{f c} @ \mathbf{f e . u c} . \mathbf{p t}}$
} 


\section{INTRODUÇÃO}

Desde 1991, que a teoria baseada nos recursos (RBV) evoluiu desde a perspetiva upstart, para uma das teorias mais proeminentes e poderosas para descrever, compreender, explicar e predizer as decisões estratégicas nas organizações (Barney, Ketchen Jr., Wright, 2011). Nas últimas décadas foram desenvolvidos vários paradigmas na tentativa de explicar a vantagem competitiva sustentável das empresas, nomeadamente os modelos apresentados por Porter (1980) e Shapiro (1989) que enfatizam o poder do mercado, os modelos de Barney (1991) e Teece, Pisano, \& Shuen, (1997) que enaltecem a eficiência da empresa, a teoria baseada nos recursos ("Resourcebased View of the Firm" - RBV), de Barney (1991), que foca as capacidades e recursos específicos da empresa e os mecanismos de isolamento como determinantes fundamentais da eficiência da empresa para criar vantagem competitiva (Rumelt, 1984; Barney, 1986, 1991; Peteraf, 1993) em Teece, Pisano, \& Shuen, (1997), e a teoria das "Capacidades Dinâmicas", uma extensão da RBV, que explora e identifica as dimensões das capacidades específicas da empresa que podem ser fonte de vantagem competitiva, para depois explicar como as combinações de competências com os recursos podem ser desenvolvidas e protegidas para a sobrevivência da empresa em envolventes de mudança (Teece, Pisano, \& Shuen, 1997).

A essência da teoria baseada nos recursos reside na ênfase dada aos recursos e às capacidades como génese da vantagem competitiva. $O$ conjunto de recursos e capacidades estão distribuídos de forma heterogénea pelas empresas concorrentes - uma condição que permite diferenças nas rendas dos recursos entre as empresas - e são imperfeitamente móveis - uma condição que permite a estas diferenças persistirem no tempo - para atingirem vantagem competitiva (Mahoney \& Pandian, 1992; Wernerfelt, 1984; Barney, 1991; Eisenhardt \& Martin, 2000; Wang e Ahmed, 2007; Leiblein, 2011).

As empresas que possuam recursos valiosos e raros podem alcançar vantagem competitiva e gozar de melhorias na performance a curto prazo. Para sustentar tais vantagens, no longo prazo, os recursos da empresa devem ser também inimitáveis e não-substituíveis, (Barney, 1991). Estas são as hipóteses baseadas na essência ou assunções da RBV, testadas por académicos (Newbert, 2008; Amit \& Schoemaker, 1993; Barney, 1991; Eisenhardt \& Martin, 2000; Henderson \& Cockburn, 1994; Powell, 2001; Teece, Pisano \& Shuen, 1997) e que permite na maioria dos autores, identificar que recurso ou capacidade específica se deve explorar, para que uma empresa alcance vantagem competitiva numa indústria em particular (Newbert, 2007, 2008). Esta abordagem metodológica utilizada pela maioria dos académicos (abordagem da heterogeneidade dos recursos) apresenta conceptualmente uma forte limitação. É necessária então uma abordagem de nível conceptual que identifique que características dos recursos e capacidades da empresa estão relacionados com a vantagem competitiva e com a performance, permitindo aos gestores que competem com estratégias alternativas às da concorrência, autonomamente identificar, pesquisar e depois explorar recursos e capacidades que contribuam não só para melhorar a sua posição competitiva mas que se ajustem aos seus modelos de negócio únicos (Newbert, 2008). De facto, para concluir que um recurso ou capacidade é valioso, raro e inimitável simplesmente porque está relacionado com a vantagem competitiva é necessário assumir que as hipóteses da RBV que ligam o valor, a raridade e a inimitabilidade dos recursos à vantagem competitiva são factuais e não necessitam de confirmação empírica. Para melhor compreender porque é que os recursos e capacidades contribuem para a vantagem competitiva da empresa, as suas características subjacentes deverão ser analisadas empiricamente (Newbert, 2008; Leiblein, 2011, p. 912). Seguimos aqui a ideia de Fernando Pessoa quando afirma que "Toda a teoria deve ser feita para poder ser posta em prática, e toda a prática deve obedecer a uma teoria" (Pessoa, 1926).

$\mathrm{O}$ nosso trabalho concentrou-se assim num estudo empírico sobre a relação direta entre as combinações valiosa, raras e inimitáveis de recursos com capacidades, a vantagem competitiva e a performance. Os resultados baseados na análise das hipóteses fundamentais da RBV a um nível conceptual, preenchem um importante gap na literatura empírica. $\mathrm{O}$ facto do valor, raridade e inimitabilidade mais do que recursos e capacidades específicos, determinarem a vantagem competitiva da empresa, fornece suporte para as hipóteses que foram na sua maioria e até agora, aceites na base da lógica e da intuição. Seguindo o estudo realizado por Scott L. Newbert, (2008), procurase testar e explorar no contexto português, os seus resultados e ultrapassar algumas das suas limitações. Também neste estudo procuramos testar a hipótese formulada por académicos que, em termos conceptuais, existem diferenças entre vantagem competitiva e performance, podendo estes deixar de ser utilizados de forma indiferenciada, e evidenciar que a vantagem competitiva tem um importante papel nos processos de exploração da combinação de recursos com capacidades. Procura-se contribuir com resultados, que permitam aos gestores decidir sobre o desenvolvimento de novas formas de combinar os recursos com capacidades a que têm acesso e deixar de procurar novos recursos e capacidades. Acrescenta-se aos atributos valor e raridade no estudo original o atributo "inimitabilidade", através da sua dimensão "ambiguidade causal" (Barney, 1991; Dierickx \& Cool, 1989). Simultaneamente, complementam-se resultados anteriores sobre o desenvolvimento da ambiguidade 
causal na inimitabilidade dos recursos e capacidades na melhoria da performance das empresas (King \& Zeithaml, 2001).

Para que os recursos e capacidades da empresa tenham um potencial de vantagem competitiva sustentável é necessário que detenham os atributos valor para explorar oportunidades e / ou neutralizar ameaças no envolvente da empresa; raridade entre os concorrentes atuais e potenciais da empresa; difíceis de imitar ou total inimitabilidade; e sem substitutos equivalentes (Barney, 1991). Os recursos da empresa podem ser considerados fontes de vantagem competitiva quando são valiosos, permitindo à empresa conceber ou implementar estratégias que aumentem a sua eficiência e eficácia, e por isso, explorarem oportunidades ou neutralizam ameaças no envolvente da empresa (Barney, 1991, p.101). Esta definição enfatiza as propriedades de eficiência de um recurso e significa o valor de um recurso ou conjunto de recursos em termos do seu ajustamento técnico ou produtividade (Leiblein, 2011, p. 912).

Uma empresa obtém vantagem competitiva sustentável quando possuir recursos e capacidade valiosos, raros, inimitáveis e não substituíveis. A sustentabilidade depende da facilidade com que esses ativos possam ser replicados. Newbert, (2007,p.138) conclui que nas últimas duas décadas a inimitabilidade (condição para a sustentabilidade da vantagem competitiva a par da não substitutabilidade) tem sido medida por diferentes dimensões (King \& Zeithaml 2001, Barney, 1991, Dierickx \& Cool, 1989, Lippman \& Rumelt, 1982, Reed \& DeFillippi, 1990, Rumelt, 1984, 1987, Irwin, Hoffman, \& Lamont, 1998). Com base nos resultados apresentados por Newbert (2007) a maioria dos testes com suporte empírico (70\%) evidenciaram que a inimitabilidade pode contribuir substancialmente para a posição competitiva e que existem diversas formas das empresas protegerem os seus recursos, capacidades e capacidades core, da imitação pela concorrência. É, no entanto, importante focar as características da dimensão ambiguidade para medir a inimitabilidade enquanto atributo mais importante dos recursos e capacidades na teoria da RBV (Newbert, 2007).

A visão da empresa baseada nos recursos é uma das perspetivas teóricas mais bem aceites no campo da gestão estratégica com argumentos amplamente divulgados nas revistas académicas (Newbert, 2007, p.121). Mas para que uma teoria seja considerada "verdadeira", i.e., que tenha um suporte empírico sobre os seus princípios centrais, dever-se-á assumir que tal teoria passou por uma avaliação empírica bem documentada. E, como refere Leiblein (2011, p.925) ao discutir os conceitos core da teoria baseada nos recursos, dos recursos estratégicos e das capacidades dinâmicas, a forma mais confiável de obter resultados empíricos de elevada qualidade, é requerer estudos elaborados de forma cuidadosa que isolem os constructos específicos de interesse. Além destas características, a teoria da gestão estratégica contemporânea está preocupada em primeiro lugar em explicar diferenças de performance entre as empresas (Newbert, 2007, p.126) pelo que é relevante testar a relação entre a variedade de variáveis independentes alternativas como a diversificação, o crescimento, as entradas em novos mercados, recursos específicos, inimitabilidade, valor, raridade, e as varáveis dependentes como a performance sustentada, vantagem competitiva, e a vantagem competitiva sustentada (Newbert, 2007,p.126).

Com o objetivo de aumentar a compreensão da RBV, Newbert (2007), optou pela revisão e análise da literatura empírica, identificou a forma como a RBV tem sido empiricamente testada e avaliou o seu nível de suporte empírico recebido. Concluiu que apesar da atenção considerável da literatura empírica, esta apenas ofereceu um nível de suporte marginal, notando que o nível de suporte empírico varia de forma considerável com a variável independente utilizada nos testes. De acordo com as abordagens teóricas testadas em cada artigo do conjunto de dados do trabalho de Newbert (2007, p.127), foi possível listar a abordagem da heterogeneidade dos recursos, a abordagem de nível conceptual, a abordagem organizacional e a abordagem das capacidades dinâmicas.

A abordagem conceptual operacionaliza a variável independente em termos do valor, raridade, inimitabilidade, e/ou não-substituibilidade dos recursos e capacidades para explicar a variação na vantagem competitiva (variável dependente). Deste modo, permitirá aos gestores identificar automaticamente, e depois pesquisar e explorar recursos e capacidades que contribuam não só para a sua posição competitiva mas que se ajustem com os seus modelos de negócio únicos (Newbert, 2008). Resultados de estudos ao nível conceptual, fornecem o conhecimento sobre as características dos recursos e capacidades que em geral as empresas deverão possuir para aumentar a sua posição competitiva. Académicos que utilizem uma abordagem ao nível conceptual para analisar o nível de suporte empírico da RBV devem procurar testar se os atributos dos recursos essenciais para que um recurso contribua efetivamente para a vantagem da empresa, são de facto prognósticos significativos para estes fins, ao invés de identificar os atuais recursos e capacidades que conferem uma vantagem para a empresa (Newbert, 2007, p.128).

\subsection{Modelo de Pesquisa Proposto}

Pretendemos analisar se e em que grau os recursos e capacidades com valor, raridade e inimitabilidade melhoram, atualmente, a posição competitiva da empresa, dando continuidade ao estudo de Newbert $(2007,2008)$ de modo a superar algumas das limitações detetadas e satisfazer o interesse de académicos e gestores. Ao testar as hipóteses da RBV, reforçamos a teoria RBV em relação a estudos anteriores, pelas seguintes formas. A primeira, analisase o atributo "inimitável" subjacente à teoria RBV 
através de duas variáveis associadas à ambiguidade causal. A segunda, analisa-se os atributos "valioso" e "raro" sobre variáveis únicas resultantes da combinação de recursos com capacidades ao invés da pesquisa que envolve de forma individual os recursos e as capacidades valiosos e raros como variável independente (Newbert, 2008,p.747). Operacionalizar a variável independente com estas características justifica-se quando consideramos que devemos medir um recurso raro em termos do conjunto de recursos possuído pelas empresas competitivas e também em termos das suas funções (Newbert, 2008:139 citando Peteraf \& Bergen,2003). Ou, como refere Penrose "os serviços produzidos pelos recursos são uma função do modo como são usados - de forma exata os mesmos recursos quando usados para diferentes propósitos ou em diferentes formas e em combinação com diferentes tipos ou conjuntos de outros recursos fornecem um serviço diferente ou conjunto de serviços (Penrose, 1959,p.25). Nestas circunstâncias parece que um dado recurso sendo raro por definição, os serviços fornecidos por esses recursos podem não o ser.
$\mathrm{Na}$ terceira forma de aproximação à teoria RBV, uma vez colocada a hipótese da vantagem competitiva mediar a relação recurso/capacidade com a performance, o estudo explora o efeito intermediário da vantagem competitiva na performance da empresa. Deste modo, para recolher resultados da performance dos seus recursos e capacidades, uma empresa deve primeiro alcançar a vantagem competitiva que resulta da exploração da combinação desses recursos e capacidades, i.e., por si só, o valor, a raridade e a inimitabilidade dos recursos e capacidades de uma empresa podem não melhorar a performance. Tal só poderá ser alcançado se a empresa for hábil em explorar a combinação desses recursos e capacidades valiosos e raros, e que lhe permita efetivamente reduzir custos, explorar oportunidades de mercado e/ou neutralizar ameaças competitivas (Newbert, 2008,p.761). Apesar da vasta literatura sobre a Teoria $\mathrm{RBV}$, a atenção da literatura empírica com respeito ao modelo por nós proposto é limitada (Figura 1).

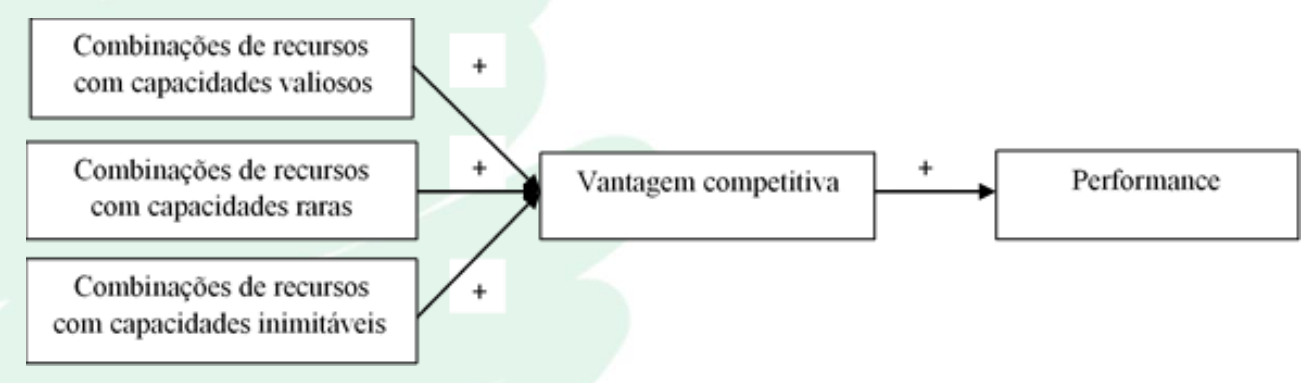

Figura 1 - Modelo de pesquisa proposto Fonte: Elaboração própria

\subsection{Hipóteses a estudar}

Barney (1991) defende que a magnitude da vantagem competitiva de uma empresa será função do valor dos seus recursos e das suas capacidades na redução dos custos e/ou na resposta às oportunidades e ameaças do envolvente, e que o alcance da vantagem competitiva se deve à habilidade na eficiência da utilização de tais recursos e capacidades. A empresa deverá ter acesso a capacidades apropriadas para explorar recursos e capacidades que estejam à sua disposição (Amit \& Schoemaker, 1993,p.35). Um uso eficiente dos recursos e das capacidades é visto em termos de possíveis combinações com outros recursos ou capacidades" (Penrose, 1995, p.86, Newbert, 2008,p.748). Para Makadok (2001), as empresas podem criar rendas pela escolha dos melhores recursos e pela exploração mais eficiente dos recursos com as capacidades apropriadas. Por isso, enquanto um recurso pode possuir um potencial valor elevado, tal valor só poderá ser realizado quando for combinado com a correspondente capacidade, ou enquanto uma capacidade pode possuir um potencial valor elevado, tal valor só poderá ser realizado quando for combinado com o correspondente recurso. Assim, quanto maior o valor da combinação de recursos com capacidades maior a vantagem como resultado da sua exploração.

Hipótese 1: o valor das combinações de recursos e capacidades que uma empresa explora está positivamente relacionado com a sua vantagem competitiva.

Barney (1991) refere que "uma empresa tem vantagem competitiva quando implementa uma estratégia que crie valor, não implementada simultaneamente por uma atual ou potencial concorrente" (Barney, 1991,p.102). Nesta perspetiva, porque os recursos e capacidades devem ser explorados em combinação, e como a raridade contribui para a vantagem competitiva, tal raridade será superior em combinações de recursos com capacidades, e não apenas ao nível individual de recursos e capacidades. Recursos (ou capacidades) comuns podem ser essenciais para o alcance de vantagem competitiva desde que façam par com uma capacidade (ou recurso) 
de uma tal forma que o resultado da exploração da sua combinação, surja raro (Newbert, 2008,p.748). Neste sentido, se a combinação de um recurso com uma capacidade que uma empresa explora é rara, então deverá alcançar vantagem competitiva.

Hipótese 2: A raridade da combinação dos recursos com capacidades que uma empresa explora está positivamente relacionada com a sua vantagem competitiva.

A inimitabilidade é o atributo da RBV mais importante (Barney, 2001; King \& Zeithaml, 2001). Os recursos valiosos e raros só podem ser considerados fonte de vantagem competitiva, se as empresas que não possuem esses recursos não os possam obter (Barney, 1991). A imitação de um recurso ou capacidade, levará tempo, custos ou ambos dependendo da facilidade relativa com que as empresas concorrentes são capazes de acumular um recurso ou capacidade semelhante. Isto é, a imitação de um ativo está relacionada com as características do processo de acumulação de ativos (Dierickx e Cool, 1989,p.1507). A ambiguidade causal ocorre quando a ligação entre o recurso possuído pela empresa e a vantagem competitiva sustentável da empresa não é compreendida ou compreendida de forma muito imperfeita pela concorrência. (Barney, 1991).

Hipótese 3: A inimitabilidade da combinação dos recursos com capacidades que uma empresa explora está positivamente relacionada com a vantagem competitiva.

Os termos "vantagem competitiva" e "performance" são frequentemente usados como possuindo o mesmo significado, mas são conceptualmente distintos (Powell, 2001). Enquanto a vantagem competitiva está normalmente conceptualizada como a implementação de novas estratégias atualmente não implementadas por outras empresas que facilitem a redução de custos, a exploração de oportunidades de mercado, e/ou a neutralização de ameaças competitivas (Barney, 1991), a performance está geralmente conceptualizada como a renda que uma empresa alcança como resultado da implementação de estratégias. Sob qualquer teoria de estratégia, a performance existe, tem causas específicas e estas causas estão diretamente relacionadas com o conceito de vantagem competitiva. A performance, e não a vantagem competitiva, deverá ser sempre a variável dependente, Powell (2001,p.876).

Nem sempre a vantagem competitiva é uma condição suficiente para melhorar a performance. Newbert (2008,p.749) indica alguns autores que comprovam empiricamente que existem fatores exógenos, que podem afetar significativamente a performance. Em circunstâncias da empresa ser incapaz de se apropriar a baixo custo do valor económico produzido, qualquer melhoria na performance que uma empresa experiencie, é improvável correlacionar perfeitamente com a sua vantagem competitiva (Newbert, 2008,p.749). Assim, enquanto é esperado que a vantagem competitiva e a performance estejam correlacionadas, os dois conceitos são teoricamente e empiricamente distintos (Newbert, 2008,p.749). Enquanto a vantagem competitiva se refere ao valor económico criado pela combinação de recursos e capacidades da empresa, a performance refere-se ao valor económico que uma empresa capturou da sua comercialização. A vantagem competitiva que uma empresa alcança é um antecedente importante da performance. Neste sentido, é expectável que a performance das empresas que sejam capazes de alcançar vantagem competitiva seja maior que a performance das empresas que não são capazes de tal feito (Newbert, 2008,p.750).

Hipótese 4: A vantagem competitiva de uma empresa está positivamente relacionada com a sua performance.

Seguindo a lógica de Barney (1991), Newbert (2008,p.750), afirma que as empresas com melhor performance são as que exploram com maior eficiência a combinação de recursos e capacidades valiosos e raros. Para melhorar a performance os atores empresariais deverão identificar e implementar estratégias baseadas nos recursos que atualmente resultam na criação de valor económico. Enquanto algumas empresas são capazes de ter acesso e de explorar recursos e capacidades potencialmente valiosos não garante a apropriação positiva de rendas económicas. Para colher benefícios de performance dos recursos e capacidades raros, valiosos e inimitáveis, a empresa deve de os empregar em combinação de modo a reduzir custos, a explorar oportunidades de mercado, e/ou a neutralizar ameaças do ambiente. A performance é função da eficiência com que se explora a combinação dos recursos com capacidades, em oposição ao valor e raridade (Newbert, 2007,p.750). Independentemente de quão raras e valiosas estas combinações são, elas não irão predizer a performance da empresa. Enquanto uma empresa possa reconhecer incapacidade em melhorar a sua performance na ausência de combinações de recursos com capacidades valiosos e raros, é a vantagem competitiva que deriva da sua exploração que determina o nível de performance de uma empresa (Newbert, 2008,p.780)

Hipótese 5: A vantagem competitiva de uma empresa medeia a relação entre $o$ valor das combinações de recursos com capacidades que a empresa explora e a sua performance.

Hipótese 6: A vantagem competitiva da empresa medeia a relação entre a raridade das combinações de recursos com capacidades que a empresa explora e a sua performance. 
Hipótese 7: A vantagem competitiva da empresa medeia a relação entre a inimitabilidade das combinações de recursos com capacidades que a empresa explora e a sua performance.

\section{METODOLOGIA}

No estudo por nós realizado utilizámos o inquérito proposto por Newbert (2008) com as adaptações e alterações necessárias à amostra de empresas de dimensões e sectores de atividade distintos a operar em Portugal. Foi também por nós introduzida a variável inimitabilidade não utilizada originalmente.

\subsection{Variáveis}

Devido à limitação da racionalidade humana por parte dos respondentes na capacidade de avaliar o valor, raridade e inimitabilidade de cada combinação de recursos com capacidades controlados pela empresa (Simon, 1978,p.356) os respondentes foram questionados sobre diversas categorias de recursos e capacidades. Neste sentido, foi utilizada a tipologia de recursos e capacidades físicas, financeiras, humanas e organizacionais de Barney (1997) e acrescentada a tipologia "recursos e capacidades intelectuais" de Newbert (2008). Os respondentes foram solicitados a avaliar o potencial em que os recursos e capacidades das categorias referidas, servem o propósito de reduzir os custos a um nível competitivo, de explorar as oportunidades de mercado esperadas, e de se defenderem sobre ameaças competitivas conhecidas.

A construção dos itens relacionados com o valor e com a raridade das combinações de recursos com capacidades e dos itens subjacentes à vantagem competitiva foi replicada de Newbert (2008). Refinando o modelo original do autor, é acrescentada a variável "ambiguidade causal" enquanto dimensão da inimitabilidade dos recursos e capacidades. Nestas circunstâncias, foram desenvolvidos quatro novos itens de medição da ambiguidade causal considerando que caso uma empresa com vantagem competitiva não compreenda a relação entre os recursos que controla e as suas vantagens, as outras não poderão aprender sobre essa relação, adquirindo os recursos necessários (assumindo que não são imperfeitamente imitáveis por outras razões) para implementar estratégias relevantes.

Semelhante à metodologia utilizada na variável valor, a ambiguidade torna-se mais elevada quando um recurso é combinado com a capacidade apropriada (lógica da função multiplicativa). Nesta lógica, a combinação de recursos com capacidades terá uma função da ambiguidade de um recurso individual com capacidades que o englobe. Estes itens estão positivamente codificados tal que quanto maior o grau de resposta, maior é a ambiguidade da combinação dos recursos com as capacidades da empresa. Em termos de cálculo dos resultados, as respostas aos itens de cada recurso desconhecido pela concorrência foram multiplicados pelos correspondentes itens de capacidades resultando num score relativo à sua combinação. Posteriormente foi calculada uma média dos 5 scores relativos ao desconhecimento sobre as estratégias da empresa por parte da concorrência na tentativa de reduzir custos a um nível competitivo, de explorar oportunidades previstas, e de neutralizar ameaças conhecidas com os recursos e capacidades a que tem acesso atualmente. A mesma lógica e metodologia foi aplicada no cálculo das respostas aos itens relativos à compreensão dos resultados alcançados pela empresa quando combina cada recurso com a respetiva capacidade. As respostas aos itens referenciados resultaram num score da combinação de cada recurso com capacidade e calculada a média dos 5 scores, resultante num valor relativo à compreensão da empresa sobre os resultados das estratégias implementadas.

No trabalho de Scott Newbert (2008), é desenvolvida a relação entre recursos e capacidades da empresa com a performance, optando este por avaliar a performance através da escala da performance de mercado (Delaney e Huselid's, 1996), escala subjetiva que inclui indicadores financeiros (vendas e rentabilidade) e não financeiros (marketing e quota de mercado), adequada a amostras de empresas privadas. Sendo a amostra deste estudo relativa a empresas privadas, é utilizada a mesma escala, onde os itens estão positivamente codificados tal que quanto maior a resposta melhor a performance da empresa. Estas variáveis são calculadas somando as respostas dos quatro itens da escala da performance de mercado.

As variáveis de controlo utilizadas seguem autores de referência na pesquisa da Teoria RBV, que tipicamente controlam pelo tamanho da empresa (número total de trabalhadores) e pela hostilidade envolvente (escala de Khandwalla, 1997). A escala utilizada para a variável performance de mercado foi a de tipo Likert com 6 pontos alinhados de muito pior a muito melhor. Nas variáveis vantagem competitiva, valor, raridade, e inimitabilidade foi utilizada uma escala de Likert de cinco pontos com itens alinhados de 1 - Totalmente em desacordo a 5 - Totalmente de acordo.

Por último, foi dada a possibilidade dos respondentes solicitarem o envio dos resultados do presente trabalho caso o solicitassem no final do inquérito. A taxa de resposta relativamente ao "Sim" foi bastante positiva e encorajadora, revelando $o$ interesse do estudo para $352(91.3 \%)$ dos respondentes. Todos os campos dos questionários eram de resposta obrigatória exceto os campos referentes ao CAE, Idade, Habilitações, Tempo de experiência (no sector, na empresa, na função e da função na empresa). 


\subsection{Trabalho de campo}

No presente estudo foi utilizada uma mailing list obtida de dados secundários externos contendo 5012 empresas, a operar em diversos sectores da atividade económica portuguesa. Com referência ao Tailored Design Method de Jon Dillbert (Newbert, 2008) foi utilizado um endereço na Internet, onde o inquérito esteve disponível para os respondentes permitindo inclusivamente o envio de um lembrete eletrónico, sugerindo a estes destinatários a conclusão do questionário.

Apesar da taxa de resposta ser bastante reduzida (7.34\%), o número de inquéritos obtidos (368) para tratamento estatístico foi superior ao triplo do estudo de Newbert (117). Para avaliar a fiabilidade dos dados foram calculados os alfas de Cronbach para cada escala usada em todo o estudo (Nunnally, 1978) com resultados favoráveis de avaliação da consistência interna. Os alfas para todas as escalas estão acima de 0.700 , sugerindo uma consistência interna das escalas, com exceção para as variáveis relativas à: "Vantagem competitiva na combinação de recursos com capacidades físicos e humanos", e "Hostilidade do envolvente".

\section{ANÁLISE E DISCUSSÃO DOS RESULTADOS}

A informação dos 368 respondentes foi utilizada para calcular as estatísticas de frequência e de correlação para as variáveis do modelo. Tal como no estudo de Newbert (2008), verifica-se uma elevada diferença entre as médias das variáveis relativas ao valor das combinações de recursos com capacidades em geral e as médias das restantes variáveis do modelo, pressupondo que um recurso é exponencialmente mais valioso quando combinado com a capacidade apropriada. A operação do fator multiplicativo entre cada uma das variáveis correspondentes aos recursos com capacidades ocorreu com a transformação das variáveis relativas aos recursos com as capacidades apropriadas.

Considerando a amostra de 368 respondentes, foi apurado o coeficiente de correlação de Pearson para as variáveis do modelo e as suas correlações são na grande maioria positivas, relativamente fortes para um nível de significância de 0.01 . Os resultados do cálculo da variável "Hostilidade do envolvente" indicam uma tendência dos respondentes na escolha de uma pontuação superior indicativa de um envolvente hostil. No cálculo do alcance da vantagem competitiva, os resultados das estatísticas descritivas indicam que as empresas da amostra, valorizam (concordam com) o acesso a combinações de capacidades com recursos físicos, financeiros, humanos, intelectuais, e organizacionais não possuídos atualmente pela empresa.

Uma análise aos resultados das combinações raras de recursos com capacidades da empresa permite identificar um valor médio superior nos recursos com capacidades Humanas, Organizacionais, Intelectuais, Físicos, e Financeiros. A estatística descritiva para a inimitabilidade das combinações de recursos com capacidades permite identificar que existe uma maior incidência de valores nas combinações de recursos com capacidades financeiras (média), Humanas e Organizacionais. As estatísticas descritivas das combinações dos recursos e capacidades desconhecidas pela concorrência indicam que a média das empresas respondentes perceciona de modo elevado que a concorrência desconhece a forma como a empresa combina recursos com capacidades intelectuais e organizacionais. Os dados utilizados da análise fatorial indicam a amostra do estudo é superior à sugerida por Tabachnick e Fidell (2007) em Pallant, 2007) que recomenda pelo menos 300 casos para a análise fatorial. Nunnally (1978) recomenda um rácio de 10:1, isto é, 10 casos para cada item. Outros indicam um rácio de 3:1 (Cattell, 1978). Newbert (2008) apresenta um rácio de 5.1:1 e o corrente estudo, um rácio de 3.5:1.Os resultados da análise fatorial do modelo atual, produziram 5 componentes principais resultantes das combinações lineares das variáveis iniciais.

Os fatores identificados são estáveis pelo que se conclui que as escalas usadas no estudo são de facto indicadores válidos dos constructos que pretendem medir. O valor da estatística KMO é de 0.870 , significando a adequação da amostra e o valor do Teste de Bartlett tem um nível de significância 0.000. A análise dos resultados da extração de fatores (Tabela 1) permite concluir que os clusters das variáveis relativas ao "valor" têm altos pesos apenas no Fator 1, fazendo parte do mesmo grupo e mostrando que existe grande correlação apenas com esse fator, pelo que o Fator 1 se vai denominar "valor". 
Análise dos Fundamentos da Teoria Baseada nos Recursos

Tabela 1 - Matriz de Componentes Principais

\begin{tabular}{|l|c|c|c|c|c|}
\hline \multirow{5}{*}{ VARIÁVEIS } & \multicolumn{5}{c|}{ COMPONENTES } \\
\cline { 2 - 6 } & $\mathbf{1}$ & $\mathbf{2}$ & $\mathbf{3}$ & $\mathbf{4}$ & $\mathbf{5}$ \\
\hline V4_Rec_explrar_oport & $\mathbf{, 8 6 1}$ &,- 176 &, 002 &,- 193 &,- 058 \\
\hline V3_Cap_explrar_oport & $\mathbf{, 8 5 3}$ &,- 216 &,- 019 &,- 155 &,- 083 \\
\hline V5_Cap_respndr_amçs & $\mathbf{, 8 3 8}$ &,- 244 &,- 054 &,- 155 &, 015 \\
\hline V2_Rec_red_custos & $\mathbf{, 8 3 8}$ &,- 175 &,- 033 &,- 158 &,- 057 \\
\hline V6_Rec_respndr_amçs & $\mathbf{, 8 3 3}$ &,- 254 &,- 034 &,- 213 &, 011 \\
\hline V1_Cap_red_custos & $\mathbf{, 7 9 7}$ &,- 035 &,- 037 &,- 117 &,- 028 \\
\hline R2_Comb_Rec_novscap &,- 287 & $\mathbf{, 8 5 4}$ &, 161 &, 120 &, 065 \\
\hline R1_Comb_cap_novsrec &,- 271 & $\mathbf{, 8 0 5}$ &, 185 &, 093 &, 133 \\
\hline R3_Comb_RecCap &,- 284 & $\mathbf{, 7 7 9}$ &, 068 &, 243 &, 028 \\
\hline I2_Empr_Compr_res_Rec &,- 175 & $\mathbf{, 5 5 6}$ &, 143 &, 505 &,- 070 \\
\hline Perf_Cresc_Vnds &,- 078 &, 057 & $\mathbf{, 9 1 6}$ &, 069 &,- 027 \\
\hline Perf_Quota_Mercd &,- 034 &, 130 & $\mathbf{, 8 8 1}$ &, 073 &,- 002 \\
\hline Perf_Lucro &,- 001 &, 083 & $\mathbf{, 8 7 0}$ &, 076 &,- 052 \\
\hline Perf_Mkt &,- 005 &, 142 & $\mathbf{, 5 0 4}$ &, 209 &, 062 \\
\hline VC2_Oprt_capitalzds &,- 237 &, 183 &, 107 & $\mathbf{, 8 1 3}$ &, 060 \\
\hline VC3_Respsts_amçs &,- 261 &, 170 &, 157 & $\mathbf{, 7 8 7}$ &, 068 \\
\hline VC1_Custs_Compttvs &,- 210 &, 087 &, 118 & $\mathbf{, 7 5 3}$ &, 009 \\
\hline I4_Emps_Cmpr_res_Cap &,- 112 &, 519 &, 124 & $\mathbf{, 5 2 5}$ &,- 026 \\
\hline Avaliação_Oprt_Env &,- 031 &, 103 &,- 028 &, 046 &, $\mathbf{8 4 0}$ \\
\hline Avaliação_Seg_Env &,- 022 &, 073 &, 032 &, 021 &, $\mathbf{8 3 7}$ \\
\hline Avaliação_Contrl_Env &,- 067 &,- 054 &,- 016 &, 006 &, $\mathbf{7 8 2}$ \\
\hline
\end{tabular}

Nota. Método de extração pelas componentes principais. Método Varimax.

Fonte: Elaborado pelos autores.

Constata-se que a variável relativa inimitabilidade (ambiguidade causal) e raridade identificam-se com o Fator 2, faz parte do mesmo grupo, dado que a associação entre elas é positiva, apesar de menor grau de associação para a variável relativa à inimitabilidade. O Fator 2 irá denominar-se de "raridade". Todas as variáveis relativas à performance encontraram correlação positiva com o Fator 3, denominado de "performance". Ao Fator 4 denominado de Vantagem Competitiva, foi associada a variável correspondente à compreensão da empresa sobre os resultados alcançados pela exploração dos recursos (inimitabilidade) e ao Fator 5 associadas as variáveis relativas à "hostilidade da envolvente".

Seguindo o nosso modelo, a Hipótese 1, a Hipótese 2 e a Hipótese 3, foram testadas cada uma individualmente, com o uso do modelo hierárquico de regressão dos mínimos quadrados ordinários, um nível para as duas variáveis de controlo e um segundo nível para cada uma das 5 categorias individuais de recursos/capacidades e para a sua média. Ao estimar a regressão para cada um dos 6 modelos das variáveis associadas ao valor das combinações dos recursos com capacidades, e individualmente para as variáveis associadas apenas à raridade das combinações dos recursos com capacidades, verificou-se que todas elas eram significativas ao nível 0.001 , o que corrobora a Hipótese 1, de que o aumento do valor das combinações de Recursos com Capacidades exploradas pelas empresas contribui positivamente para a vantagem competitiva, e corrobora a Hipótese 2 , de que o aumento do valor ou grau da raridade de recursos com capacidades contribui positivamente para a vantagem competitiva.

Realce para os resultados do F-Change tanto para as variáveis valor como para as variáveis raridade, sugerindo que a introdução individual de cada uma destas variáveis, produzem um modelo que se ajusta significativamente muito melhor aos dados que o modelo das variáveis de controlo. Em relação às 
variáveis de controlo, constata-se que a variável associada ao Tamanho da Empresa ("Emprgds_categs") é insignificante para 5 dos 6 modelos.

Os valores estimados na regressão para as duas variáveis da ambiguidade causal, uma para cada um dos 6 modelos associados à inimitabilidade das combinações de recursos com capacidades produzem resultados muito significativos $(\mathrm{p}<0.001)$ para todos os modelos, no entanto apenas para a variável referente à "Empr_Cmpr_Comb_RC": Empresa compreende combinações de recursos com capacidades (Tabela 2 ).

Tabela 2 - Determinantes da Vantagem Competitiva Associada à Inimitabilidade

\begin{tabular}{|c|c|c|c|c|c|c|}
\hline \multirow[t]{2}{*}{ VARIÁVEIS } & \multicolumn{2}{|c|}{$\begin{array}{l}\text { RECURSOS E CAPACIDADES } \\
\text { FÍSICAS }\end{array}$} & \multicolumn{2}{|c|}{$\begin{array}{l}\text { RECURSOS E CAPACIDADES } \\
\text { FINANCEIRAS }\end{array}$} & \multicolumn{2}{|c|}{$\begin{array}{c}\text { RECURSOS E CAPACIDADES } \\
\text { HUMANAS }\end{array}$} \\
\hline & BLOCO 1 & BLOCO 2 & BLOCO 1 & BLOCO 2 & BLOCO 1 & BLOCO 2 \\
\hline Host. do envolvente & .017 & -.001 & .041 & .012 & .064 & .050 \\
\hline Emprgds_categs & .044 & .019 & .086 & $.097 *$ & -.008 & .022 \\
\hline EmprCmprComb_RC & & $.435 * * *$ & & $.431 * * *$ & & $.416 * * *$ \\
\hline ConcDescComb_RC & & .034 & & .030 & & 0.67 \\
\hline R2 Ajustado & -.003 & .189 & .004 & .197 & -.001 & .189 \\
\hline$F$ - Estatístico & .405 & $22.436 * * *$ & 1.684 & $23.689 * * *$ & .768 & $22.360 * * *$ \\
\hline$F$-Change & & $44.371 * * *$ & & $44.907 * * *$ & & $43.771 * * *$ \\
\hline \multirow{3}{*}{ VARIÁVEIS } & & & & & & \\
\hline & \multicolumn{2}{|c|}{$\begin{array}{l}\text { RECURSOS E CAPACIDADES } \\
\text { INTELECTUAIS }\end{array}$} & \multicolumn{2}{|c|}{$\begin{array}{l}\text { RECURSOS E CAPACIDADES } \\
\text { ORGANIZACIONAIS }\end{array}$} & \multicolumn{2}{|c|}{$\begin{array}{l}\text { MÉDIA DOS RECURSOS E } \\
\text { CAPACIDADES }\end{array}$} \\
\hline & BLOCO 1 & BLOCO 2 & BLOCO 1 & BLOCO 2 & BLOCO 1 & BLOCO 2 \\
\hline Host. do envolvente & .059 & .066 & $.154 * *$ & $.140 * *$ & $.087+$ & .068 \\
\hline Emprgds_categs & -.079 & -.060 & .017 & .022 & 0.13 & .017 \\
\hline EmprCmprComb_RC & & $.464 * * *$ & & $.436^{* * *}$ & & $.509 * * *$ \\
\hline ConcDescComb_RC & & -.016 & & .001 & & -.011 \\
\hline R2 Ajustado & .004 & .210 & 0.19 & .206 & .002 & .254 \\
\hline$F$ - Estatístico & 1.774 & $25.368 * * *$ & 4.481 & 24.800 & 1.418 & $32.197 * * *$ \\
\hline$F$-Change & & $48.501 * * *$ & & $44.062 * * *$ & & $62.499 * * *$ \\
\hline
\end{tabular}

Nota. Reportados os Coeficientes Standardizados $+\mathrm{p}<0.10,{ }^{*} \mathrm{p}<0.05, * * \mathrm{p}<0.01, * * * \mathrm{p}<0.001$

Fonte: Elaborado pelos autores.

Este resultado parece confirmar que será mais forte a inimitabilidade quando a empresa não perceciona as combinações de recursos com capacidades utilizadas pela empresa para o alcance da vantagem competitiva, ou que para medir a inimitabilidade das combinações de recursos com capacidades deverá ser utilizada apenas uma das duas variáveis da ambiguidade causal. Esta segunda sugestão vai ao encontro de Barney (1991) quando refere que a ambiguidade causal é uma de duas causas e não das duas em simultâneo. Neste sentido poderá afirmar-se que os resultados da regressão aqui utilizados vêm comprovar empiricamente e ao nível conceptual, o âmbito da ambiguidade causal a que Barney se refere, o que corrobora a Hipótese 3 de que o aumento da inimitabilidade da combinação de recursos com capacidades contribui positivamente para o alcance da vantagem competitiva. Estas conclusões iniciais abrem boas perspetivas para o modelo proposto que conjuga o valor, a raridade e a inimitabilidade como determinantes da vantagem competitiva.

Ao introduzir no $1^{\circ}$ modelo (das variáveis de controlo) as variáveis relativas ao valor e raridade, os correspondentes parâmetros estimados são positivos e significantes para todos os modelos de recursos com capacidades ao nível 0.001 , oferecendo resultados com suporte empírico muito superiores comparados com os resultados de Newbert (2008). Utilizando o mesmo modelo de regressão hierárquica para os 6 modelos de recursos com capacidades, os resultados referentes à introdução ao primeiro modelo (variáveis de controlo) das variáveis valor, raridade e da variável da ambiguidade causal, Hipótese 1, Hipótese 2 e Hipótese 3 em simultâneo (Tabela 3). Uma vez mais, os resultados de F-estatístico e F-Change são significativos sugerindo que o modelo global se ajusta aos dados e que a adição de diferentes variáveis independentes produz modelos que se ajustam aos dados de forma significativamente melhor que o controlo das variáveis. Os modelos globais explicam uma quantidade considerável da variância na vantagem competitiva tendo feito crescer o modelo das variáveis de controlo. Para o modelo com a categoria média dos recursos/capacidades e na interpretação do coeficiente de determinação ajustado, $\mathrm{R}^{2}$, as variáveis independentes "Hostilidade do envolvente", "Emprgds_categs", "valor", "raridade", "Emp_Compr_Comb_RC", contribuem ou explicam em média cerca de $3 \overline{5} \%$ da variabilidade da vantagem competitiva. Os parâmetros das variáveis de controlo mostram que a hostilidade do envolvente é apenas 
Análise dos Fundamentos da Teoria Baseada nos Recursos

significante para o modelo de recursos com capacidades organizacionais $(\mathrm{p}<0.1)$ e a variável
"Emprgds_categs" é significante no modelo dos recursos com capacidades financeiras.

Tabela 3 - Determinantes da Vantagem Competitiva com Base no Modelo

\begin{tabular}{|c|c|c|c|c|c|c|}
\hline \multirow[b]{2}{*}{ VARIÁVEIS } & \multicolumn{2}{|c|}{$\begin{array}{c}\text { RECURSOS E CAPACIDADES } \\
\text { FÍSICAS }\end{array}$} & \multicolumn{2}{|c|}{$\begin{array}{l}\text { RECURSOS E CAPACIDADES } \\
\text { FINANCEIRAS }\end{array}$} & \multicolumn{2}{|c|}{$\begin{array}{c}\text { RECURSOS E CAPACIDADES } \\
\text { HUMANAS }\end{array}$} \\
\hline & BLOCO 1 & BLOCO 2 & BLOCO 1 & BLOCO 2 & BLOCO 1 & BLOCO 2 \\
\hline Host. do envolvente & .017 & -.018 & .041 & -.012 & .064 & .015 \\
\hline Emprgds_categs & .044 & .007 & .086 & $.111 *$ & -.008 & .029 \\
\hline Valor & & $.160^{* *}$ & & $.122 *$ & & $.187 * * *$ \\
\hline Raridade & & $.107+$ & & $.181 * *$ & & $.227 * * *$ \\
\hline Emp_Compr_Comb_RC & & $.316^{* * * *}$ & & $.309 * * *$ & & $.264 * * *$ \\
\hline R2 Ajustado & -.003 & .225 & .004 & .242 & -.001 & .271 \\
\hline$F$ - Estatístico & .405 & $22.284 * * *$ & 1.684 & $24.403 * * *$ & .768 & $28.263 * * *$ \\
\hline$F$-Change & & $36.791 * * *$ & & $39.197 * * * *$ & & $46.401 * * *$ \\
\hline \multirow[t]{2}{*}{ VARIÁVEIS } & \multicolumn{2}{|c|}{$\begin{array}{l}\text { RECURSOS E CAPACIDADES } \\
\text { INTELECTUAIS }\end{array}$} & \multicolumn{2}{|c|}{$\begin{array}{c}\text { RECURSOS E CAPACIDADES } \\
\text { ORGANIZACIONAIS }\end{array}$} & \multicolumn{2}{|c|}{$\begin{array}{c}\text { MÉDIA DOS RECURSOS E } \\
\text { CAPACIDADES }\end{array}$} \\
\hline & BLOCO 1 & BLOCO 2 & BLOCO 1 & BLOCO 2 & BLOCO 1 & BLOCO 2 \\
\hline Host. do envolvente & .059 & .025 & $.154 * *$ & $.096^{*}$ & $.087+$ & .026 \\
\hline Emprgds_categs & -.079 & -.044 & .017 & .024 & .013 & .023 \\
\hline Valor & & $.242 * * *$ & & $.180 * * *$ & & $.234 * * *$ \\
\hline Raridade & & $.281 * * *$ & & $232 * * *$ & & $.178 * *$ \\
\hline Emp_Compr_Comb_RC & & $.236 * * * *$ & & $.265 * * *$ & & $.325 * * *$ \\
\hline R2 Ajustado & .004 & .350 & .019 & .290 & .002 & .346 \\
\hline$F$ - Estatístico & 1.774 & $40.457 * * *$ & $4.481 *$ & $31.036^{* * * *}$ & 1.418 & $39.765 * * *$ \\
\hline$F$-Change & & $65.616^{* * * *}$ & & $47.596 * * *$ & & \\
\hline
\end{tabular}

Nota: Reportados os Coeficientes Standardizados. $+\mathrm{p}<0.10,{ }^{*} \mathrm{p}<0.05, * * \mathrm{p}<0.01, * * * \mathrm{p}<0.001$

Fonte: Fonte: Elaborado pelos autores.

Os parâmetros estimados para a variável relativa à ambiguidade causal (inimitabilidade) são, para todas as 6 categorias de recursos/capacidades, positivos e significativas ao nível 0.001 , o que leva, mais uma vez, a corroborar a Hipótese 3 de que a inimitabilidade da combinação dos recursos com capacidades que uma empresa explora está positivamente relacionada com a vantagem competitiva, significando que quanto maior a inimitabilidade da combinação de recursos com capacidades da empresa maior o alcance da vantagem competitiva. De notar que no teste à Hipótese 3 está referenciada apenas a compreensão da empresa sobre a relação entre a combinação dos recursos com capacidades que controla e as suas vantagens. Considerando tais resultados é possível afirmar que a ambiguidade causal é de facto uma dimensão da inimitabilidade contribuindo positivamente para $o$ alcance da vantagem competitiva das empresas.

\subsection{Determinantes da performance}

Para testar a Hipótese 4 foram utilizados os 6 modelos hierárquicos de regressão descrito antes. Os 6 $F$-estatísticos são significantes, sugerindo que o modelo global se ajusta aos dados. A mudança nos $6 F$ estatísticos, com a introdução da variável "Vantagem Competitiva" no modelo, melhora o ajustamento do modelo, com significância, apenas em 4 dos 6 modelos (os modelos dos recursos com capacidades financeiras e humanas não são significativos). Houve um incremento de $5.5 \%$ para $13 \%$ ao longo dos 6 modelos, pelo que a introdução da variável "Vantagem Competitiva" aumentou o modelo das variáveis de controlo, contribuindo significativamente como determinante da performance de mercado. 
Tabela 4 - Determinantes da Performance de Mercado

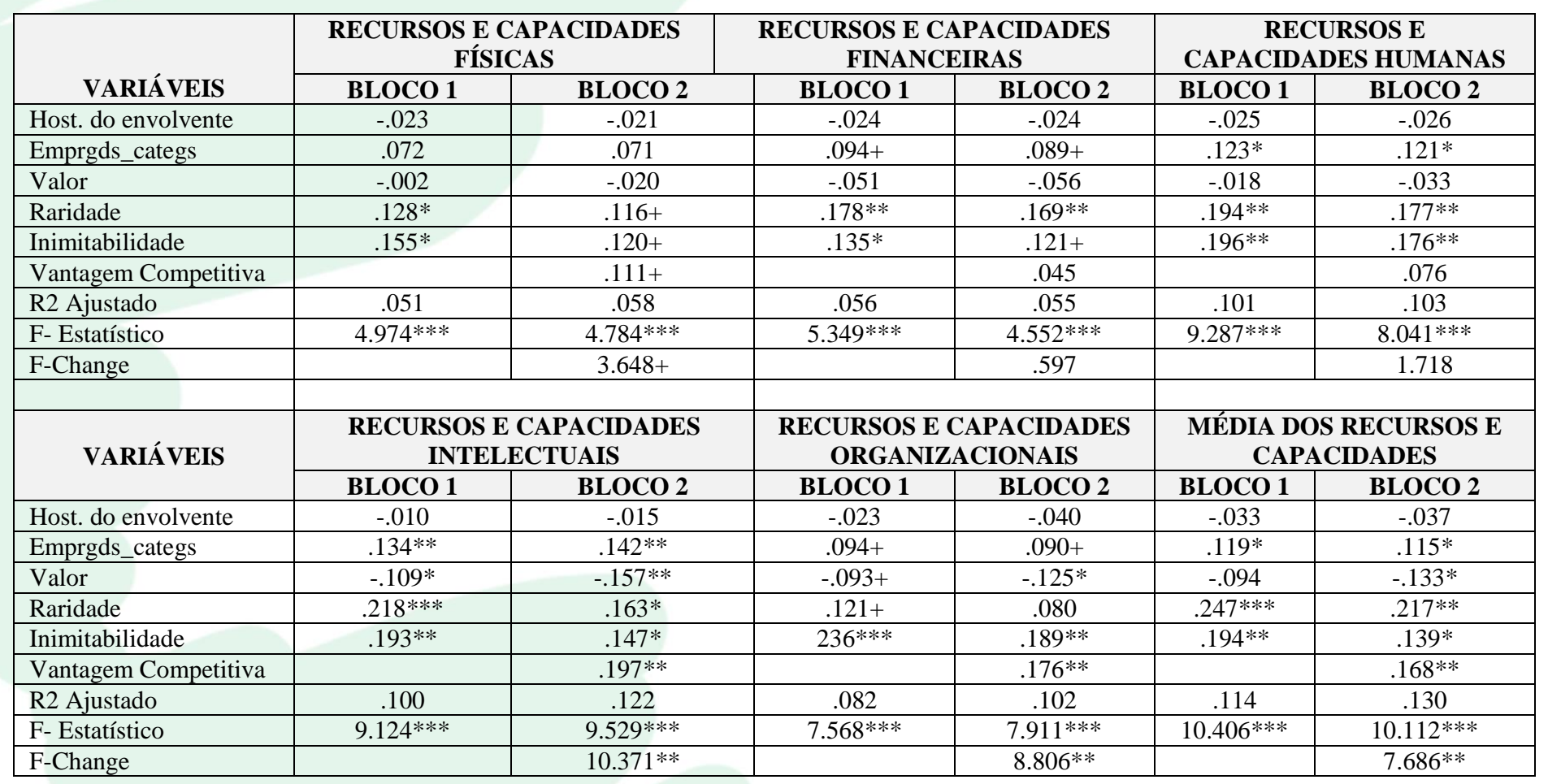

Nota. Reportados Coeficientes standardizados $-+\mathrm{p}<0.10, * \mathrm{p}<0.05, * * \mathrm{p}<0.01, * * * \mathrm{p}<0.001$

Fonte: Fonte: Elaborado pelos autores.

Os parâmetros estimados para as variáveis de controlo mostram que a hostilidade da envolvente não é significativa, mas negativamente relacionada com a performance em todos os 6 modelos, sugerindo que quanto menor a hostilidade do envolvente de uma empresa, maior a sua performance. Os parâmetros estimados para o tamanho da empresa são significativos para todos os modelos, sugerindo que esta variável se encontra relacionada com a performance, com exceção do modelo de recursos e capacidades físicas. Relativamente aos parâmetros associados à variável valor, todos os parâmetros apresentam resultados negativos incluindo os modelos significativos nomeadamente os modelos de recursos e capacidades intelectuais, organizacionais e o modelo da média dos recursos e capacidades.

Os parâmetros associados às variáveis raridade e inimitabilidade são significantes para os seis modelos (com exceção do parâmetro da variável raridade no modelo associado aos recursos e capacidades organizacionais). Tais resultados significam que tanto a raridade como a inimitabilidade contribuem positivamente como determinantes da performance. Resultados semelhantes são encontrados em 4 dos 6 modelos de regressão para a vantagem competitiva, nomeadamente para os modelos recursos e capacidades físicos, intelectuais, organizacionais e para a média dos modelos. Tais resultados permitem corroborar a Hipótese 4 de que a vantagem competitiva está positivamente relacionada com a performance e por isso a vantagem competitiva é um antecedente importante na performance

O efeito mediador da vantagem competitiva. Considerando as referências a Baron e Kenny (1986) em Newbert (2008), sobre as considerações analíticas para a mediação, deverão seguir-se 4 condições para concluir sobre a Hipótese 5, a Hipótese 6 e a Hipótese 7. Os resultados observados permitem satisfazer a primeira condição: o valor, a raridade e a inimitabilidade das combinações estão relacionados com a vantagem competitiva.

A Tabela 4, evidencia ainda que o nível de significância para os parâmetros valor, raridade e inimitabilidade são reduzidos ou eliminados depois da inclusão ao modelo da variável vantagem competitiva, pelo que a quarta condição de Baron e Kenny (1986) é satisfeita. Numa análise global da Hipótese 5, da Hipótese 6 e da Hipótese 7 é possível sugerir que a vantagem competitiva medeia em parte as relações entre o valor e a performance (no modelo dos recursos e capacidades intelectuais, organizacionais e a média dos recursos/capacidades); em parte, as relações entre a raridade e a performance para todos os modelos com exceção do modelo de recursos e capacidades organizacionais; e de forma global as relações entre a inimitabilidade e a performance em todos os modelos. 
Para avaliar o efeito mediador da vantagem competitiva na relação do valor, raridade e inimitabilidade com a performance de forma rigorosa, foram aplicados os testes Sobel, Aroian e Goodman, desenhados para determinar se a influência de uma variável mediadora numa relação de uma variável independente com uma variável dependente é significativa. Os resultados do nosso estudo mostram a significância de cada um dos testes estatísticos, e sugerem que o efeito mediador da vantagem competitiva é de facto significativo. Estes resultados superam largamente os alcançados por Newbert (2008) contribuindo de forma muito positiva para o conhecimento do efeito mediador da vantagem competitiva em relação ao valor, raridade e inimitabilidade com a performance.

\section{CONCLUSÕES}

As empresas tomam decisões estratégicas com base na análise de informação externa e interna à organização. Uma análise interna à organização incide sobre os recursos e capacidades físicas, financeiras, humanas, intelectuais e organizacionais. A teoria baseada nos recursos da empresa (TBR) enfatiza os recursos e capacidades valiosos e raros como fonte de vantagem competitiva. Para sustentar tais vantagens, os recursos e capacidades deverão ser inimitáveis e nãosubstituíveis. Estas são as hipóteses testadas por académicos, baseadas na essência da TBR e que têm contribuído para um contínuo interesse na exploração do conhecimento sobre a gestão estratégica das organizações. A análise da literatura empírica permitiu identificar a relevância atual do campo de gestão estratégica contemporâneo em explicar as diferenças de performance entre as empresas com base nas variáveis independentes alternativas como a diversificação, o crescimento, recursos específicos, inimitabilidade, valor, raridade, e as varáveis dependentes como a performance sustentada, vantagem competitiva, e vantagem competitiva sustentada (Newbert, 2007:126).

Surge na literatura um reduzido nível de suporte empírico no teste das assunções da teoria baseada nos recursos quando a variável independente é operacionalizada como um recurso específico comparando com quando a variável independente é operacionalizada como valor. $\mathrm{O}$ estudo aqui apresentado seguiu o modelo de investigação de Newbert (2008), e tem como objetivo medir as hipóteses da RBV, nomeadamente que o valor, a raridade e a inimitabilidade das combinações de recursos com capacidades da empresa contribuem para o alcance da vantagem competitiva e esta, por sua vez, contribui para a sua performance. Para o efeito foi utilizada uma abordagem conceptual para analisar que características de recursos e capacidades da empresa estão relacionados com a vantagem competitiva e/ou performance. Esta abordagem, introduz os atributos valor, raridade, inimitabilidade, e/ou não-substituivel dos recursos e capacidades como variável independente para explicar a variação na variável dependente, vantagem competitiva. Concebendo a variável independente em termos de combinações de recursos com capacidades (em oposição aos recursos ou capacidades individuais), este trabalho contribui fortemente para uma melhor perceção da dinâmica pela qual os recursos e capacidades têm servido de argumento em contribuírem para a vantagem competitiva do que a maioria da pesquisa anterior nesta área. Ao utilizar uma abordagem ao nível conceptual para análise do nível de suporte empírico da RBV, os académicos procuram testar se os atributos essenciais dos recursos que contribuem efetivamente para a vantagem da empresa são de facto prognósticos significativos para tal alcance, ao invés de identificar quantidades de atuais recursos e capacidades específicos correlacionando-os com a vantagem competitiva, como utilizado frequentemente na abordagem heterogénea.

Com interesse em complementar o modelo proposto e anteriormente descrito, foi introduzida uma nova Hipótese no sentido de testar a relação da inimitabilidade (através da ambiguidade causal) da combinação dos recursos com capacidades, com a vantagem competitiva, tendo contribuído para a expansão do conhecimento empírico sobre os mecanismos de isolamento protetores da imitação da combinação de recursos com capacidades da empresa e do desenvolvimento da ambiguidade causal na inimitabilidade dos recursos e capacidades na melhoria da vantagem competitiva das empresas.

A amostra de referência foi distinta face à amostra utilizada no estudo de Newbert (2008) nomeadamente, o âmbito de abrangência da pesquisa foi transversal às atividades económicas existentes em Portugal. $\mathrm{O}$ inquérito utilizado resultou numa amostra de 368 inquéritos favoráveis ao aprofundamento do estudo. A amostragem utilizada representa 97,8\% de empresas com menos de 250 trabalhadores, sendo mais de metade $(56 \%)$ microempresas, $29,3 \%$ pequenas empresas e $12,5 \%$ médias empresas, oriundas da indústria transformadora $(19,7 \%)$, comércio por grosso e retalho $(12,2 \%)$ e atividades de consultoria, científica e técnicas similares $(11,1 \%)$.

As Hipóteses 1, Hipótese 2 e Hipótese 3 foram testadas, e os seus resultados contribuem com um dos poucos estudos com evidência empírica da relação direta entre valor, raridade e inimitabilidade das combinações dos recursos com capacidades da empresa e a sua vantagem competitiva. A análise aos resultados destas três Hipóteses confirmam suporte empírico e contribuem em muito para o preenchimento de uma importante falha na literatura empírica da teoria baseada nos recursos até agora aceite na maioria das vezes pela lógica e intuição: o valor, a raridade e a inimitabilidade da combinação de recursos com capacidades, mais do que os recursos e capacidades 
específicos, determinam a vantagem competitiva da empresa. Com o presente trabalho foi possível corroborar empiricamente a hipótese de que a vantagem competitiva está positivamente relacionada com o nível de performance obtido. Além deste importante contributo, é possível afirmar que para obter ganhos derivados da performance a empresa deverá primeiro alcançar vantagem competitiva que deriva da combinações valiosas, raras e inimitáveis de recursos com capacidades. Estes resultados enriquecem a aceitação da teoria baseada nos recursos como teoria rigorosa da gestão estratégica.

\section{CONTRIBUTOS E RECOMENDAÇÕES}

Este estudo empírico aumentou o campo do conhecimento da estratégia ao analisar o valor, a raridade e inimitabilidade de combinações de recursos com capacidades que permitem obter melhor performance por intermédio da vantagem competitiva. Tal conhecimento permitirá aos gestores explorar os atuais recursos e capacidades em combinação de modo a orientar esforços para os recursos e capacidades que mais impacto têm no seu modelo de negócio ao invés de explorar os recursos e capacidades específicos de uma determinada indústria.

Sendo um dos poucos estudos a testar empiricamente as características valor, raridade dos recursos e capacidades bem como a ambiguidade causal associada à combinação de recursos com capacidades da empresa com contributos para a vantagem competitiva, futuros estudos poderão incidir o seu objeto de análise, na relação das variáveis associadas à ambiguidade causal com a performance da empresa. Ao aprofundar esta temática poderá ser explorado o grau de importância ou interesse das empresas em percecionar as combinações de recursos com capacidades utilizadas para o alcance da vantagem competitiva ou em percecionar o que a concorrência conhece ou desconhece acerca dessas mesmas combinações.

Propomos no futuro testar empiricamente o valor, raridade e inimitabilidade das combinações de recursos com capacidades associadas a outras indústrias para além da indústria transformadora, o comércio por grosso e retalho e atividades de consultoria aqui testadas, de modo a compreender se existem diferenças substanciais no modo como as empresas de determinada indústria definem as suas estratégias com referência aos atributos dos recursos e capacidades da empresa. Considerando que o atributo inimitabilidade está associado à vantagem competitiva sustentável, seria relevante estudar ao nível conceptual a hipótese de que a inimitabilidade é um fator determinante para a vantagem competitiva sustentável. Os resultados alcançados permitem corroborar a hipótese formulada por académicos no que respeita, em termos conceptuais, das diferenças entre vantagem competitiva e performance, deixando estes termos de serem utilizados de forma indiferenciada.

\section{REFERÊNCIAS}

Amit, R. \& Schoemaker, P. (1993). Strategic assets and organizational rent. Strategic Management Journal, Vol. 14, 33-46.

Barney, J.B. (1991). Firm resources as sustained competitive advantage. Journal of Management, Vol 17, No 1, 99-120.

J., Ketchen Jr., \& D. Wright, M., (2011). The future of resource-based theory: rivatalization or decline?, Journal of Management, Vol. 37 No. 5, September, 1299-1315.

Baron, R.M., \& Kenny, D.A. (1986). The moderatormediator variable distinction in social psychological research: conceptual, strategic and statistical considerations. Journal of Personality and Social Psychology 51, 1173:1182.

Cattell RB. (1978). The scientific use of factor analysis. Plenum: New York.

Delaney J.T., \& Huselid, M.A. (1996). The impact of human resource management practices on perceptions of organizational performance. The Academy of Management Journal, Vol.39, No. 4., 949-969.

Dierickx, Ingemar, \& Cool, Karel (1989). Asset stock accumulation and sustainability of competitive advantage. Management Science, Vol 35, No 12, 1504-1511.

Eisenhardt, K., \& Martin, J. (2000). Dynamic capabilities: what are they?. Strategic Management Journal, Vol 21, 1105-1121.

Henderson, R. \& Cockburn, I. (1994). Measuring competence? Exploring firm effects in pharmaceutical research. Strategic Management Journal, 15 (Winter Special Issue),63-84.

Irwin, J.G., Hoffman, J.J., \& Bruce. T. (1998). The effect of the acquisition of technological innovations. Journal of Engineering \& Technology Management, Vol. 15 (1): 25-55.

Khandwalla PN. (1976). Some top management styles, their context, and performance. Organization and Administrative Sciences, 7(4): 21-51.

King, A. \& Zeithaml, C. (2001). Competences and firm performance: examining the causal ambiguity 
Análise dos Fundamentos da Teoria Baseada nos Recursos

paradox. Strategic Management Journal, n. ${ }^{\circ} 22,75-$ 99.

Leiblein, M. (2011, July). What do resource-and capability-based theories propose?, Journal of Management, Vol. 37 № 4, 909-932.

Lippman, Steven A. \& Rumelt, Richard P. 1982. Uncertain imitability: an analysis of inter firm deficiency under competition. The Bell Journal of Economics, 13: 418-438.

Mahoney, Joseph \& Pandian, Rajendran (1992). The resource-based view within the conversation of strategic management. Strategic Management Journal, Vol 13, 363-380.

Makadok, (2001). Toward a synthesis of the resourcebased and dynamic-capability views of rent creation. Strategic Management Journal; Vol.22. 387-401.

Newbert, S.L. (2007). Empirical research on the resource-based view of the firm: an assessment and suggestions for future research. Strategic Management Journal; Vol. 28. 121-146.

S.L. (2008). Value, rareness, competitive advantage, and performance: a conceptual-level empirical investigation of the resource-based view of the firm. Strategic Management Journal, Jan, 29, 745-768.

Nunnally, JC. (1978). Psychometric theory. (2nd ed.). New York: McGraw-Hill Book Company.

Pallant, J. (2007). SPSS survival manual: a step by step guide to data analysis using SPSS for windows (Version 15). ( $3^{\text {rd }}$ edition). Allen\&Unwin.

Penrose, E. (1995). The theory of the growth of the firm. (3 rd Edition). Oxford University Press.

Pessoa, Fernando (1926). Palavras iniciais. Revista de Comércio e Contabilidade, $\mathrm{n}^{\mathrm{o}}$ 1, p.5
Peteraf, M.A. (1993). The cornerstones of competitive advantage: A resource-based view. Strategic Management Journal,Vol.14, 179-191.

Porter, Michael E. (1980). Competitive Strategy. New York: The Free Press.

Powell, T.C. (2001). Competitive advantage: logical and philosophical considerations. Strategic Management Journal, Vol.22, 875-888.

Reed, R., DeFillippi, R.j., (1990). Causal ambiguity, barriers to imitation, and sustainable competitive advantage. Academy of Management Review, 15, 88-102.

Rumelt, Richard P. (1984). Towards a Strategic Theory of the Firm. in Richard B. Lamb, ed. Competitive Strategic Management. New Jersey: Englewood Cliffs.

Richard. P. (1987).Theory, strategy, and entrepreneurship. in D. J. Teece (ed.). The Competitive challenge: Strategies for industrial innovation and renewal. 137-158. Cambridge, MA: Ballinger.

Shapiro, C.(1989). The theory of business strategy. RNAD Journal of Economics, Vol.20, 125-137

Simon, (1978). Rational decision-making in business organizations. Economic Sciences, Nobel Memorial Lecture, 8, 351-357.

Teece, D., Pisano, G., \& Shuen, A. (1997). Dynamic capabilities and Strategic management. Strategic Management Journal, Vol 18:7, 509-533

Wang, C. \& Ahmed, P. (2007). Dynamic capabilities: a review and research agenda. International Journal of Management Reviews, Volume 9, Issue 1, 31-55.

Wernerfelt, B. (1984). A Resource-based view of the firm. Strategic Management Journal, Vol 5, 171180 